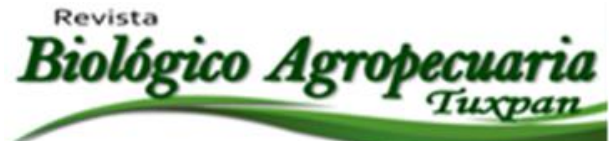

\title{
Análisis financiero y económico del cultivo del pistache en el Municipio de López, Chihuahua
}

Financial and economic analysis of the pistache crop in the Municipality of López, Chihuahua

De León Delgado M. M., Legarreta González M. A. ${ }^{\circledR}$, Olivas García J. M., Guerrero Morales S., Baray Guerrero M. R.

Universidad Autónoma de Chihuahua, Facultad de Ciencias Agrícolas y Forestales

${ }^{\square}$ Autor para correspondencia: mlegarre@uach.mx

Recibido: 12/07/2020

Aceptado: 15/08/2020

\section{RESUMEN}

El cultivo del pistachero (Pistacia vera L.) se inició en el estado de Chihuahua en los años 90’s, en donde se implementaron huertos en los municipios de Meoqui, Ojinaga, Casas Grandes, Salaices y Delicias, donde presentan potencial de productividad con desarrollado satisfactorio. Los objetivos fueron analizar la factibilidad de la inversión económica y financiera de la producción de pistache en el rancho "El último esfuerzo", en el municipio de López, Chihuahua, así como identificar los equipos y demás insumos requeridos, e investigar instancias de gobierno que pudieran apoyar el proyecto. Se utilizó la metodología de Baca Urbina, con indicadores como el VAN, TIR, B/C. Se encontró que existe un nicho de oportunidad para el cultivo en la región; es una especie rústica, de un manejo agronómico sencillo, y los costos de implantación son alcanzables. La inversión más grande a realizar es la instalación de riego por micro aspersión, así como la inversión en material vegetal. Requiere una inversión inicial de $\$ 182,491.28$, y presenta un retorno de inversión a partir del noveno año, ya que es un proyecto a largo plazo. Una vez realizado el análisis de rentabilidad, se encontró que el proyecto tiene una VAN de $\$ 208,250.86$, TIR de $14.66 \%$ y una Relación B/C de 1.76, por lo cual es factible.

Palabras clave: Agronegocios, indicadores, costos, secano, ambiente.

\begin{abstract}
The cultivation of pistachio (Pistacia vera L.) started in the state of Chihuahua in the 90's, where orchards were implemented in as the municipalities of Meoqui, Ojinaga, Casas Grandes, Salaices and Delicias, where they have potential of productivity with satisfactory development. The objectives were to analyze the feasibility of the economic and financial investment of the pistachio production in the ranch "El Último Esfuerzo", located at the municipality of López, Chihuahua, as well as to identify the equipment and other required inputs, and to investigate government agencies that could support the project. The Baca Urbina methodology was used, with indicators such as NPV, IRR, B / C. It was found that there is a niche of opportunity for cultivation in the region; It is a rustic species, with a simple agronomic
\end{abstract}


management, and the implantation costs are achievable. The largest investment to be made is the installation of micro-sprinkler irrigation, as well as investment in plant material. It requires an initial investment of $\$ 182,491.28$, and presents a return on investment from the ninth year, since it is a longterm project. Once the profitability analysis was carried out, it was found that the project has a NPV of $\$ 208,250.86$, IRR of $14.66 \%$ and a B / C Ratio of 1.76, for which it is feasible.

Keywords: agribusiness, indicators, costs, dry land, environment

\section{INTRODUCCIÓN}

El pistacho o pistache, es el fruto producido del árbol pistachero (Pistacia vera L), el cual se desarrolla endémicamente en las regiones cálidas y secas del Medio Oriente, el Mediterráneo y América (ABIDI, 2016). Pertenece a la familia de las Anacardiaceae, género Pistacia (con cuatro secciones: Lentiscella, Eu lentiscus, Butmela y $\mathrm{Eu}$ terebintus). El género está constituido por 11 especies, siendo la P. vera (Eu terebintus), la única especie con frutos de importancia económica (Lavín A., Reyes M., \& Almarza D., 2014).

El origen, comercialización y aprovechamiento de este cultivo proviene de distintos países que conjugan el Medio Oriente como lo son Irán, Israel, Turquía, Siria y Afganistán. A nivel local, en los años 90’s se establecieron parcelas gracias a la Dirección de Desarrollo Rural del Gobierno del Estado de Chihuahua con el apoyo del Sistema de Educación Tecnológica Agropecuaria, en regiones como lo son Meoqui, Ojinaga, Casas Grandes, Salaices y Delicias, donde los huertos que fueron atendidos han presentado un potencial de productividad y se han desarrollado de manera satisfactoria (González Zuccolotto \& González de las Casas, 2012).

La adaptabilidad de este cultivo al estado posee diversas ventajas como el que, históricamente, el precio por kilogramo de pistacho es más alto en comparación a la nuez pecanera (cultivo altamente producido en la zona sur del estado y el cual requiere una elevada cantidad de agua). $\mathrm{Su}$ cosecha se da durante el mes de agosto, 2 meses antes que el nogal pecanero, y posee mayor rendimiento por hectárea después de los 6 años de producción (González Zuccolotto \& González de las Casas, 2012). Además, tiene un menor impacto medioambiental, debido al bajo consumo de agua, ya que cuenta con un sistema radicular capaz de alcanzar grandes profundidades, lo que lo convierte en un árbol resistente a climas áridos, permitiéndole desarrollarse en suelos alcalinos, calcáreos y salinos (Valseco, 2009).

El pistachero es un árbol dioico, esto quiere decir que posee especímenes masculinos y femeninos (Lavín A. , Reyes M., \& Almarza D., 2014) y requiere del viento para el traslado $\mathrm{y}$ polinización de las hembras, por lo que es de gran importancia la dirección dominante del viento. La proporción de los polinizadores en una plantación debe aproximarse al 12\% (8:1, esto quiere decir que, por cada 8 hembras, debe existir un polinizador) (Saez Sanchez, 2017).

El pistachero es una especie que florece a finales del mes de marzo, en caso de las variedades tempranas y, en caso de las variedades tardías, éstas florecen a mitad del mes de abril. A partir de ese punto, es crucial que no se alcancen 
temperaturas por debajo de $\operatorname{los}-2^{\circ} \mathrm{C}$ el cultivo necesita la suficiente acumulación de unidades de calor desde el mes de abril hasta septiembre, para así obtener una óptima maduración de los frutos (Saez Sanchez, 2017).

De acuerdo con el Centro de Información de Estadística del Estado (CIEE), el municipio de López, Chihuahua consta de un clima semiárido extremoso, con una temperatura máxima de hasta $41.7^{\circ} \mathrm{C}$ y una mínima de $-14.1^{\circ} \mathrm{C}$. Presenta una temperatura media anual de $18.3^{\circ} \mathrm{C}$. Cuenta con una precipitación pluvial media anual de 363.9 milímetros, un promedio anual de 61 días de lluvia y una humedad relativa del 48\% (CIEE, 2016).

Al momento de planearse el establecimiento de un huerto pistachero es necesaria la correcta elección del material vegetal que se utilizará para la zona, ya que la longevidad estimada de un árbol pistachero, en plena producción, es de 100 años, por lo que es importante conocer las características de las variedades a escoger y del patrón que se utilizará para la plantación (Martínez San Miguel, 2014).

Se pretende impulsar la implantación de un cultivo diferente. Ante las perspectivas de un calentamiento global y falta de agua, el pistachero es un cultivo que se adapta bien, debido a su origen desértico, con pocas necesidades de agua, así como la necesidad de frío en invierno.

El objetivo general del estudio es analizar la factibilidad de la inversión económica y financiera de la producción de pistache en el rancho "El Último Esfuerzo", en el municipio de López, Chihuahua, así como investigar diferentes instancias de gobierno que pudieran apoyar para la producción de pistache.

Dentro de las expectativas de producción, se espera que la producción se incremente conforme aumenta la edad, registrando producciones de 1 a $5 \mathrm{~kg}$ a partir de los 6 años de edad, culminando en una producción promedio de $10-15 \mathrm{~kg}$ de fruto por árbol a partir de los 10 años de edad, con una expectativa de vida de 100 años del árbol (Cruzat G. \& Bachler R, 2010).

\section{MATERIALES Y MÉTODOS}

Las variables evaluadas fueron los costos de producción y costos de infraestructura.

Los indicadores utilizados para precisar la viabilidad del proyecto son el Valor Actual Neto (VAN), Tasa Interna de Rendimiento (TIR) y la Relación Beneficio/Costo (B/C) (Baca Urbina, 2010).

Se incluye la primera parte del proceso, la cual abarca desde la plantación, hasta que es cosechado y entregado a un tercero como fruto fresco.

Dentro de la siguiente Figura 1, aparece un círculo rojo, representando la localización del pozo más cercano al terreno; así como un recuadro amarillo, muestra el lugar exacto del huerto, a ubicar en las coordenadas 26.986022 105.167276 


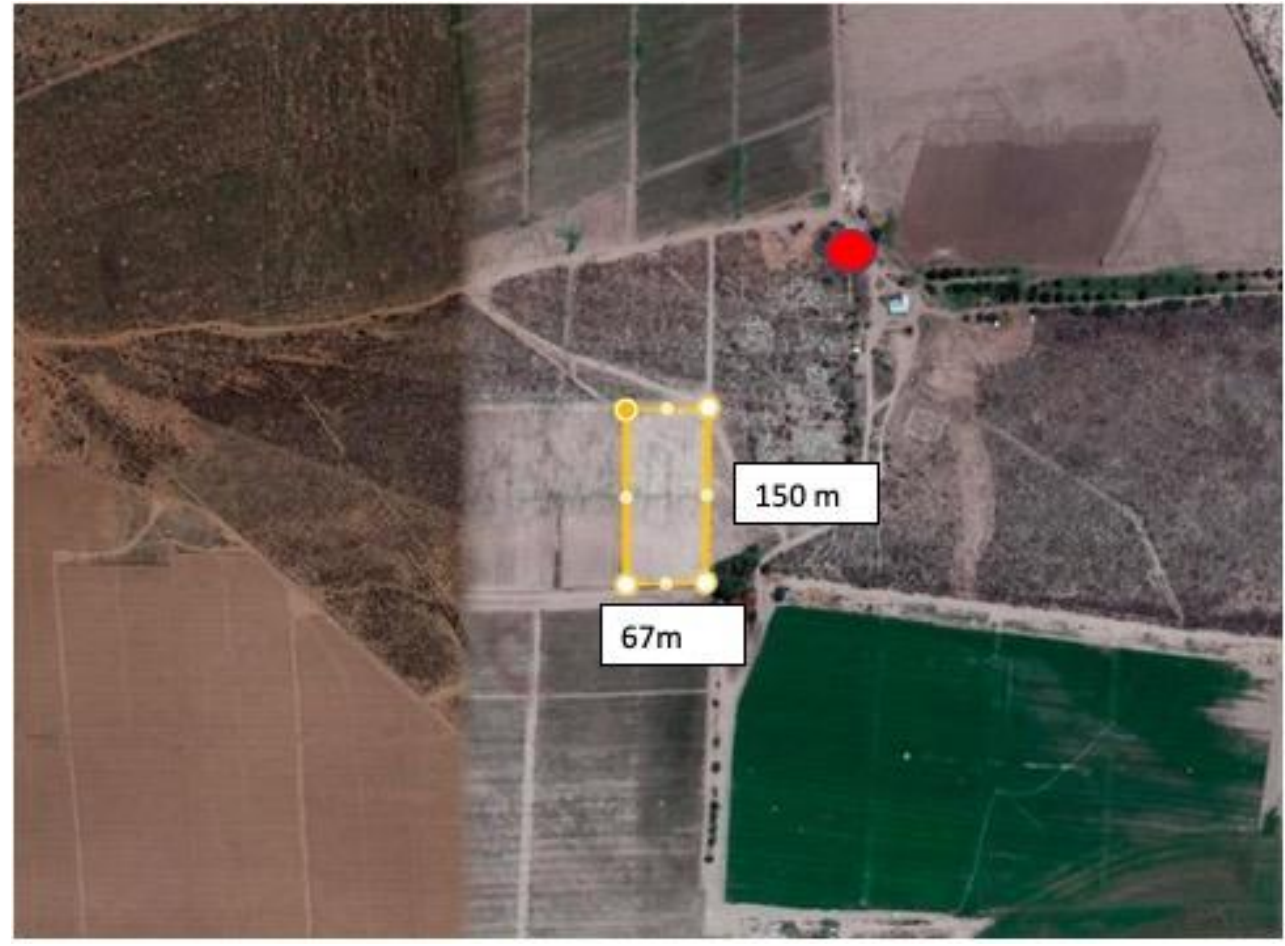

Figura 1. Ubicación tentativa del huerto.

\section{RESULTADOS Y DISCUSIÓN}

Producción de pistache en la región sur del estado de Chihuahua.

Cumpliendo con el primer objetivo, el cual consiste en la realización de un diagnóstico sobre la producción del pistache en la región sur del estado de Chihuahua, se encontró que en la región actualmente existen alrededor de 63 ha de pistachero splantadas, las cuales se distribuyen en los municipios de Jimenez, Parral, Valle de Allende y El Tule.
Maquinaria y equipo

En relación con el segundo objetivo, el cual consiste en determinar los materiales y maquinaria requeridos para el huerto; en el Cuadro 1 se presentan las labores necesarias para la preparación del suelo, para establecer la huerta, así como los costos de dichas prácticas. Se incluye desde el subsoleo hasta la nivelación del mismo. Ya que el huerto se cotizó para una hectárea, no es opción el adquirir maquinaria propia, y es por eso que se optó por el alquiler del servicio.

Cuadro 1. Preparación del terreno para el establecimiento de una hectárea de pistachero en el municipio de López, Chih.

\begin{tabular}{lll}
\hline \multicolumn{2}{l}{ Preparación para la siembra } \\
\hline Concepto & \multicolumn{2}{l}{ Costo (M.N.) } \\
\hline Subsoleo & $\$$ & $2,000.00$ \\
\hline Barbecho & $\$$ & $2,000.00$ \\
\hline Rastreo & $\$$ & $1,000.00$ \\
\hline Nivelación & $\$$ & $1,000.00$ \\
\hline TOTAL & $\$$ & $6,000.00$
\end{tabular}


En el Cuadro 2 se presenta la cotización estimada del material vegetal a utilizar. Se decidió utilizar como porta injerto la especie $\mathrm{P}$. integerrima, debido a que en el Centro de Investigación de Recursos Naturales (CIReNa), localizado dentro del municipio de López,
Chihuahua, se han desarrollado durante años una serie de investigaciones sobre adaptación de variedades, donde se ha encontrado que dicha especie es una de las que muestra mejor adaptación y producción exitosa.

Cuadro 2. Material vegetal para el establecimiento de una hectárea de pistachero en el municipio de López, Chih.

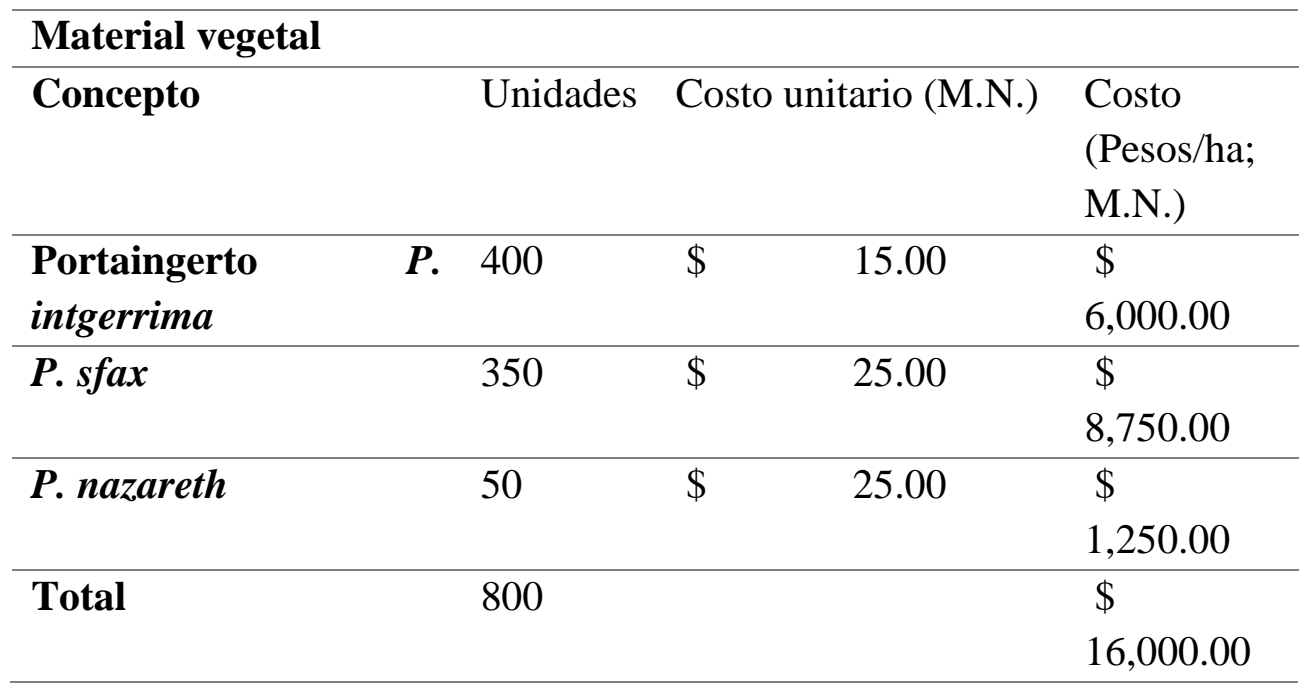

Utilizando el tipo de cambio del peso frente al dólar, al momento del estudio, el cual era de 19.90 MXN, el costo estimado de la instalación de riego por micro aspersión es de 51,751.28 pesos mexicanos.

En la Figura 2 se representa un plano donde se observa la localización del huerto, así como el detalle de los atraques, el cuerpo de válvulas y la conexión de micro aspersor. El espaciamiento entre líneas y entre emisores es de $5 \mathrm{~m}$.

El tiempo o duración de riego es aproximadamente de 3 horas, con un intervalo de 1 día entre riego, gastando aproximadamente $3.98 \mathrm{~mm} /$ día. 
De León et al., 2020

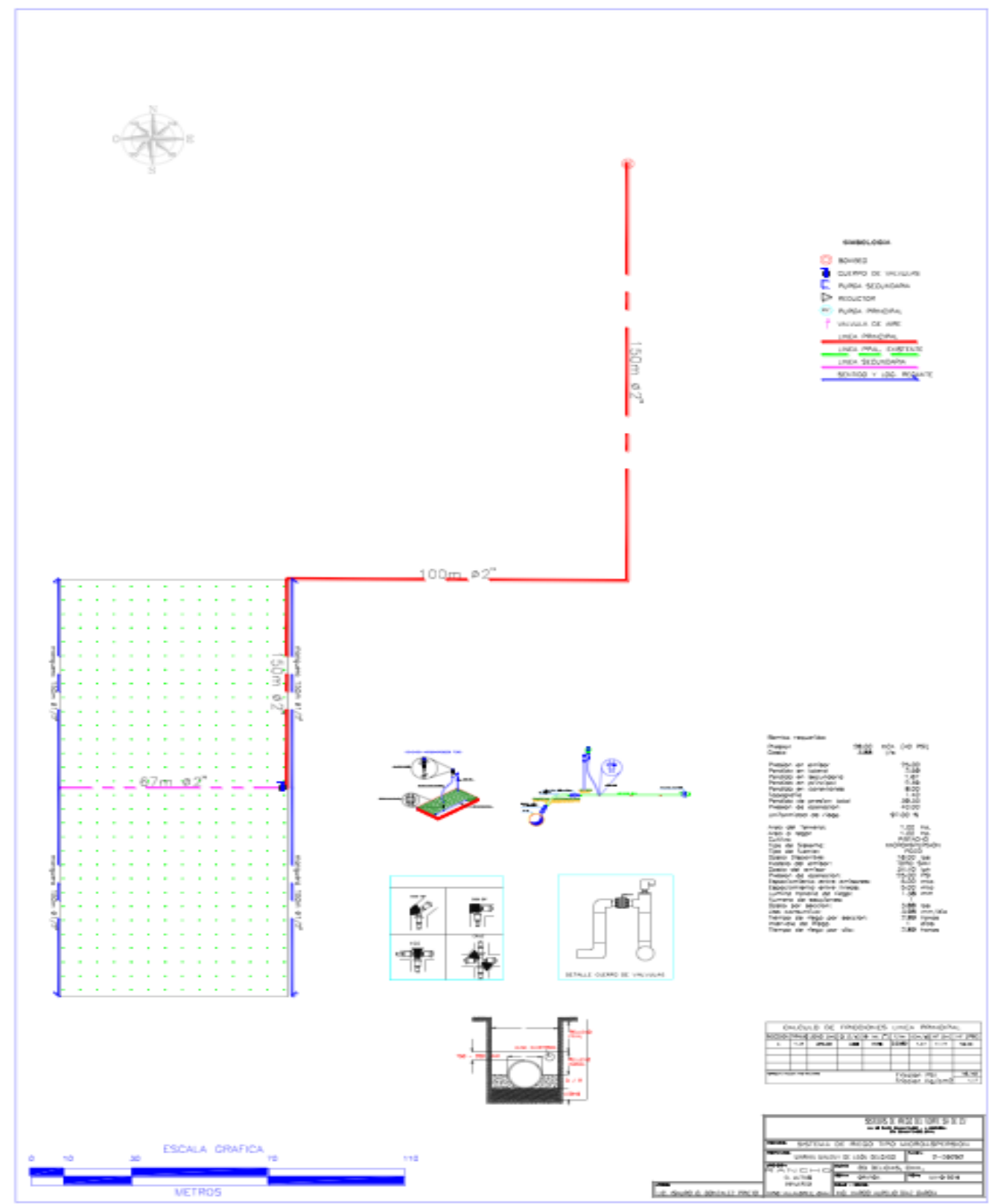

Figura 2. Plano de riego de una hectárea de pistachero en el municipio de López, Chih. Fuente: Ing. Marco Díaz. Rentabilidad potencial.

Considerando el tercer objetivo, en el cual se analizó la rentabilidad de la huerta, utilizando el paquete Microsoft Excel, se realizó una corrida financiera, utilizando costos de actividades agrícolas proporcionada por SAGARPA. Se obtuvo como inversión inicial $\$ 182,491.28$, dentro de la cual se incluyen los costos de terreno, material vegetal, instalación de riego por micro aspersión, plantación y mantenimiento del huerto, asi como los gastos administrativos durante el primer año. Esto se muestra en el Cuadro 4. Se utilizó un incremento del $5 \%$ en las proyecciones de los siguientes años. 
Cuadro 4. Costos totales de la implementación del huerto de pistachero en el municipio de López, Chih.

COSTOS TOTALES

\begin{tabular}{|c|c|c|c|c|c|c|c|c|c|c|c|c|c|c|c|c|c|c|c|c|}
\hline COSTOSFINOS & & ANNO1 & & $\mathrm{ANNO} 2$ & & AÑO3 & & ANO 4 & & AÑO5 & & AÑO6 & & ANO07 & & ANO 8 & & ANO9 & & ANO 10 \\
\hline COMPOSTA & $\$$ & $9,000.00$ & $\$$ & $9,450.00$ & $\$$ & $9,922.50$ & s & $10,418.63$ & $\$$ & $10,939.56$ & $\$$ & $11,486.53$ & s & $12,060.86$ & $\$$ & $12,663.90$ & $\$$ & $13,297.10$ & s & $13,961.95$ \\
\hline TERRENO & $\$$ & $70,000.00$ & & & & & & & & & & & & & & & & & & \\
\hline PLANTACION & $\$$ & $8,000.00$ & & & & & & & & & & & & & & & & & & \\
\hline MATERIAL VEGETAL & & $16,000.00$ & & & & & & & & & & & & & & & & & & \\
\hline RIEGO MICROASPERSION & $\$$ & $51,751,28$ & & & & & & & & & & & & & & & & & & \\
\hline RIEGOS AL AÑO & $\$$ & 624.00 & $\$$ & 655.20 & $\$$ & 687.96 & $s$ & 722.36 & $\$$ & 758.48 & $\$$ & 796.40 & $\$$ & 836.22 & $\$$ & 878.03 & $\$$ & 921.93 & s & 968.03 \\
\hline \begin{tabular}{|lll} 
OONTROL DE & PLAGAS, \\
MALEZASYENF. & \\
\end{tabular} & s & 316.00 & $\$$ & 331.80 & $\$$ & 348.39 & s & 365.81 & $\$$ & 384.10 & s & 403.30 & s & 423.47 & $\$$ & 444.64 & s & 466.88 & $\$$ & 490.22 \\
\hline FERTILIZACIÓN & $\$$ & $4,800.00$ & $\$$ & $5,040.00$ & $\$$ & $5,292.00$ & $\$$ & $5,556.60$ & s & $1,116.00$ & $s$ & $1,117.00$ & $s$ & $1,118.00$ & $\$$ & $1,119.00$ & $s$ & $1,120.00$ & $s$ & $1,121.00$ \\
\hline SUELDO ING. & $\$$ & $10,000.00$ & s & $5,000.00$ & $\$$ & $5,250.00$ & s & $7,500.00$ & $\$$ & $10,000.00$ & $\$$ & $10,500.00$ & $\$$ & $11,025.00$ & $\$$ & $11,576.25$ & s & $12,155.06$ & $s$ & $12,762.82$ \\
\hline DIVERSOS & $\$$ & $12,000.00$ & $\$$ & $12,600.00$ & $\$$ & $12,852.00$ & s & $13,109.04$ & $\$$ & $13,371.22$ & \$ & $13,638.65$ & $\$$ & $13,911.42$ & $\$$ & $14,189.65$ & $\$$ & $14,473.44$ & $\$$ & $14,762.91$ \\
\hline TOTAL & & $182,491.28$ & $\$$ & $33,077.00$ & $\$$ & $34,352.85$ & $\$$ & $37,672.43$ & $\$$ & $36,569.35$ & $\$$ & $37,941.88$ & $\$$ & $39,374.97$ & 19 & $40,871.47$ & $\$$ & $42,434,41$ & $\$$ & $44,066.93$ \\
\hline
\end{tabular}

\begin{tabular}{|c|c|c|c|c|c|c|c|c|c|c|c|c|c|c|c|c|c|c|c|}
\hline COSTOS VARIABLES & & & & & & & & ANO 4 & & ANOO5 & & $4 N 06$ & & ANN07 & & ANO 8 & & AÑO9 & ANO 10 \\
\hline COSECHA & $\$$ & . & \$ & . & $\$$ & . & s & $2,600.00$ & $\$$ & $2,730.00$ & s & $3,800.00$ & s & $5,000.00$ & s & $5,200.00$ & s & $12,000.00$ & $\$ \quad 13,000.00$ \\
\hline TRANSPORTE & & & & & & & $\$$ & $1,000.00$ & $\$$ & $1,050.00$ & $\$$ & $2,000.00$ & $\$$ & $2,600.00$ & $\$$ & $2,800.00$ & $\$$ & $3,000.00$ & $3,500.00$ \\
\hline TOTAL & $\$$ & . & $\$$ & . & $\$$ & . & $\$$ & $3,600.00$ & $\$$ & $3,780,00$ & $\$$ & $5,800.00$ & $\$$ & $7,600.00$ & $\$$ & $8,000.00$ & $\$$ & $15,000.00$ & $\$ 16,500.00$ \\
\hline
\end{tabular}

\begin{tabular}{|c|c|c|c|c|c|c|c|c|c|c|c|c|c|c|c|c|c|c|c|c|}
\hline & & AÑO1 & & AÑ 2 & & $\mathrm{ANNO}_{3}$ & & AÑO 4 & & AÑO5 & & AÑO6 & & AÑO7 & & AÑO 8 & & ANOO9 & & WNO 10 \\
\hline COSTOS FIJOS & s & $182,491.28$ & s & $33,077,00$ & s & $34,352.85$ & s & $37,672.43$ & $\$$ & $36,569.35$ & & $37,941.88$ & s & $39,374.97$ & s & $40,871.47$ & s & $42,434.41$ & s & $44,066.93$ \\
\hline COSTOS VARIABLES & $\$$ & $\cdot$ & $s$ & $\cdot$ & $\$$ & $\cdot$ & $\$$ & $3,600.00$ & & .00 & & $5,800.00$ & $\$$ & $7,600.00$ & s & $8,000.00$ & s & $15,000.00$ & s & $16,500.00$ \\
\hline COSTOS TOTALES & s & 182,491.28 & s & $33,077.00$ & s & $34,352.85$ & s & $41,272.43$ & s & $40,349.35$ & & $43,741.88$ & s & $46,974.97$ & s & $48,871.47$ & s & $57,434.41$ & s & $60,566.93$ \\
\hline
\end{tabular}

De acuerdo con diversas acopiadoras de nuez de la región, el costo promedio del de pistachero fresco es de alrededor de 90 pesos por kilogramo.

En cuanto a los ingresos proyectados para la huerta, se estima que la producción de esta comienza a partir del cuarto año, siendo el momento en el que comienzan a muestrear los árboles y comienza su producción con un estimado de $160 \mathrm{~kg}$ por hectárea.

Conforme transcurre el tiempo, la producción aumenta, llegando a producir hasta un promedio de 3,200 kg por hectárea. En el Cuadro 5 se observa una proyección del estimado de ingresos de la huerta a través de los años.

Cuadro 5. Proyección de ingresos de una hectárea del huerto de pistachero en el municipio de López, Chih.

\begin{tabular}{|c|c|c|c|c|c|c|c|c|c|c|c|}
\hline \multicolumn{12}{|l|}{ Unidades de venta } \\
\hline Presentación/Año & \multicolumn{2}{|l|}{ Año 1} & Año 2 & Año 3 & Año 4 & Año 5 & Año 6 & Año 7 & Año 8 & Año 9 & Año 10 \\
\hline Pistache (kg) & \multicolumn{2}{|l|}{-} & - & - & 160 & 160 & 416 & 800 & 2,080 & 2,880 & 3,200 \\
\hline \multicolumn{12}{|l|}{ Precio por kg (mxn) } \\
\hline Presentación/Año & \multicolumn{2}{|l|}{ Año 1} & Año 2 & Año 3 & Año 4 & Año 5 & Año 6 & \begin{tabular}{|l} 
Año \\
7
\end{tabular} & \begin{tabular}{|l} 
Año \\
8 \\
\end{tabular} & \begin{tabular}{|l} 
Año \\
9 \\
\end{tabular} & Año 10 \\
\hline Pistache $(\mathrm{kg})$ & \multicolumn{2}{|l|}{-} & - & - & 90 & 94.5 & 99.23 & 104.19 & 109.4 & 114.87 & 120.61 \\
\hline & & & & & & & & & & & \\
\hline \multicolumn{12}{|c|}{ Ingresos tentativos por año } \\
\hline \multirow{2}{*}{ Presentación/Año } & \multirow{2}{*}{ Año 1} & \multirow{2}{*}{ Año 2} & Año & Año & Año & Año & Año & Año & Año & Año & \\
\hline & & & 3 & 4 & 5 & 6 & 7 & 8 & 9 & 10 & \\
\hline Pistache (kg) & - & - & - & 14,400 & 15,120 & $41,277.60$ & 83,349 & $227,542.77$ & $330,812.18$ & $385,947.54$ & \\
\hline
\end{tabular}


Una vez realizada la corrida financiera, se utilizó una tasa de interés del 10\%, y se concluyó que el retorno de efectivo se presenta a partir del noveno año del huerto. Se obtuvo una TIR de
$14.66 \%$, una VAN de $\$ 208,250.86$ y una Relación B/C de 1.76, como se muestra en el Cuadro 6.

Cuadro 6. Rentabilidad de una hectárea de pistachero en el municipio de López, Chih.

\begin{tabular}{|c|c|c|c|c|c|c|c|c|c|c|c|c|c|}
\hline AÑo & \multicolumn{2}{|r|}{ INGRESOS } & \multicolumn{2}{|c|}{ COSTOS } & \multicolumn{2}{|c|}{ FLUJODE } & TASA & \multicolumn{2}{|c|}{ INGRESOS } & \multicolumn{2}{|c|}{ EGRESOS } & \multicolumn{2}{|c|}{ RETORNO } \\
\hline AÑO 2 & s & $=$ & $\$$ & $33,077.00$ & $-\$$ & $33,077.00$ & 0.82645 & $\$$ & - & $\$$ & $27,336.36$ & $-\$$ & $225,828.28$ \\
\hline AÑO 4 & s & $14,400.00$ & s & $41,272.43$ & $-S$ & $26,872.43$ & 0.68301 & s & $9,835.39$ & $\$$ & $28,189.63$ & $-\$$ & $287,053.56$ \\
\hline AÑO 5 & s & $15,120.00$ & s & $40,349.35$ & $-S$ & $25,229.35$ & 0.62092 & s & $9,388.33$ & $\$$ & $25,053.77$ & $-\$$ & $312,282.92$ \\
\hline AÑO 6 & $s$ & $41,277.60$ & $\$$ & 741.88 & $-s$ & $2,464.28$ & 0.56447 & $s$ & $23,300.13$ & $\$$ & $24,691.15$ & $-\$$ & $314,747.20$ \\
\hline AÑO 8 & $s$ & $227,542.77$ & s & $48,871.47$ & $\$$ & $178,671.30$ & 0.40051 & s & $106,150.38$ & $\$$ & $22,798.90$ & $-\$$ & $99,701.87$ \\
\hline AÑO 9 & 3 & $330,812.18$ & $\$$ & $57,434.41$ & $\$$ & $273,377.77$ & 0.42410 & $s$ & $140,296.66$ & $\$$ & $24,357.80$ & $\$$ & $173,675.90$ \\
\hline AÑO 10 & $s$ & $385,947.54$ & $\$$ & $60,566.93$ & $\$$ & $325,380.62$ & 0.39554 & $\$$ & $148,799.49$ & $\$$ & $23,351.17$ & $\$$ & $499,056.52$ \\
\hline TOTAL & $\$$ & $1,098,449.10$ & $\$$ & $331,542.92$ & $\$$ & $509,316.52$ & & $\$$ & $480,541.59$ & $\$$ & $272,290.73$ & & \\
\hline
\end{tabular}

Apoyo gubernamental

Programa Especial de Financiamiento de Activos Fijos para Cultivos Perennes y Proyectos con Periodos de Larga Maduración, a cargo de FIRA (Fideicomisos Instituidos en Relación con la Agricultura).

El objetivo principal del siguiente financiamiento es el promover la capitalización y modernización de las empresas, en empresas con proyectos que cuentan con un periodo de larga maduración, para de esta manera impulsar la productividad y la competitividad. Además, de adaptar la recuperación de los financiamientos a la generación de flujos de efectivo de las empresas y cubrir el riesgo de los intermediarios financieros, así como impulsar la inversión fija.

Los financiamientos deben estar respaldados por una previa evaluación técnica-financiera que determine su rentabilidad y viabilidad, la duración de la etapa pre productiva, así como las fechas y capacidad de pago.

Pro sostenible: programa de apoyo a proyectos sostenibles, a cargo de FIRA

El programa de apoyo a proyectos sostenibles es un programa especial de FIRA, el cual fue creado como apoyo financiero de los acreditados que cumplan con los objetivos de este programa, como lo son el desarrollo sostenible y el esfuerzo para mitigar el cambio climático. Dentro de los objetivos del programa, están el facilitar el acceso al crédito, a proyectos de inversión en el sector agropecuario, forestal y pesquero, que se desarrollen en el medio rural y generen beneficios al medio ambiente.

Se define como un proyecto sostenible a aquel que hace uso de los recursos naturales sin sobreexplotarlos y sin contaminarlos, respetando de esta manera ciclos naturales. Un proyecto se puede reconocer como sostenible si es economicamente rentable, socialmente justo, y ambientalmente equilibrado. Ademas, un proyecto puede reconocerse como sostenible debido a sus contribuciones a la mitigación y adaptación al cambio climático.

Con base en la información de los anteriores programas, se considera que el cultivo de pistachero tiene potencial para ser apoyado por los mismos.

\section{CONCLUSIÓN}

A través del presente estudio es posible concluir lo siguiente: 
El pistachero es una especie noble que posee grandes ventajas para ser considerado una estrategia de alternativa para productores. Es una especie rústica, de un manejo agronómico sencillo, los costos de implantación son alcanzables, no exige unidades mínimas de explotación, y es relativamente sano.

La inversión inicial del proyecto es de $\$ 182,491.28$. Siendo el sistema de riego por micro aspersión el costo más elevado en la inversión.

Debido a que es un proyecto a largo plazo, el retorno de inversión se presenta a partir del noveno año del huerto.

Una vez que el huerto se encuentra en el $100 \%$ de producción, los ingresos anuales son de aproximadamente $\$ 385,947.54$.

Es posible concluir que el proyecto es económica y financieramente aceptable. Con una TIR de $14.66 \%$, una VAN de $\$ 208,250.00$, y una Relación B/C de 1.76.

Existen planes de financiamiento que pueden apoyar la inversión, como el programa especial de financiamiento de activos fijos para cultivos perennes y proyectos con periodos de larga maduración, a cargo de FIRA.

\section{LITERATURA CITADA}

Abidi, W. (2016). Pomological and physical attributes of pistachio (Pistacia vera L.) varieties grown in west-central Tunisia.

\section{JOURNAL OF NEW SCIENCES.}

Baca Urbina, G. (2010). Evaluacion de proyectos. México, D.F.: McGraw-Hill.

Centro d información de Estadística del Estado. (2016). CIEE, Centro de Información de Estadística del Estado.

Cruzat G., R., \& Bachler R, L. (2010). Producción de Pistacho en Zonas de Secano.

González Zuccolotto, I., \& González de las Casas, H. (2012). MANUAL DEL CULTIVO DEL PISTACHERO.

Lavín A., A., Reyes M., M., \& Almarza D., P. (2014). Pistacho (Pistacia vera). Frutales de Nuez, cinco alternativas no tradicionales para el secano interior del Maule.

Martínez San Miguel, A. (2014). Plantación de pistacho de 14 Ha en el término municipal de Santa Lucía de Ocón. Universidad de La Rioja.

Saez Sanchez, I. (2017). El cultivo del pistacho. Jaen, España: Degusta Jaen .

Valseco. (2009). Cultivo moderno del pistachero. Torrebesses-(Lleida)ESPAÑA.: Casa Sant Roc. 
De León et al., 2020

Copyright (c) 2020 M. M. De León Delgado, Martín Alfredo Legarreta González, Jesús Miguel Olivas García, S. Guerrero Morales y M. R. Baray Guerrero

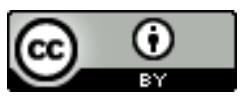

Este texto está protegido por una licencia CreativeCommons $\underline{4.0}$.

Usted es libre para Compartir — copiar y redistribuir el material en cualquier medio o formato-y Adaptar el documento —remezclar, transformar y crear a partir del material - para cualquier propósito, incluso para fines comerciales, siempre que cumpla la condición de:

Atribución: Usted debe dar crédito a la obra original de manera adecuada, proporcionar un enlace a la licencia, e indicar si se han realizado cambios. Puede hacerlo en cualquier forma razonable, pero no de forma tal que sugiera que tiene el apoyo del licenciante o lo recibe por el uso que hace de la obra.

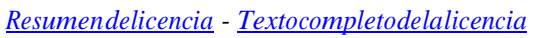

